University of Wollongong

Research Online

Faculty of Social Sciences - Papers (Archive) Faculty of Arts, Social Sciences \& Humanities

$1-1-2018$

\title{
Are carer attitudes toward medications related to self-reported medication adherence amongst people with mental illness?
}

Frank P. Deane

University of Wollongong, fdeane@uow.edu.au

Elizabeth McAlpine

University of Wollongong

Mitchell K. Byrne

University of Wollongong, mbyrne@uow.edu.au

Esther Davis

University of Wollongong, eld458@uowmail.edu.au

Christine Mortimer

University Of Wollongong, cem768@uowmail.edu.au

Follow this and additional works at: https://ro.uow.edu.au/sspapers

Part of the Education Commons, and the Social and Behavioral Sciences Commons

Research Online is the open access institutional repository for the University of Wollongong. For further information contact the UOW Library: research-pubs@uow.edu.au 


\title{
Are carer attitudes toward medications related to self-reported medication adherence amongst people with mental illness?
}

\author{
Abstract \\ Medication nonadherence among consumers with psychiatric disorders can significantly affect the health \\ and wellbeing of the consumer and their family. Previous research has suggested that carers have an \\ impact on consumer attitudes toward medication and adherence. Yet, how carer attitudes toward \\ medication may be related to consumer attitudes and adherence has received little investigation. This \\ exploratory study aimed to investigate the relationships between carer and consumer attitudes toward \\ medication and consumer adherence behaviour. A cross-sectional survey assessing consumer and carer \\ attitudes toward medication and consumer adherence was conducted amongst 42 consumer-carer \\ dyads. Correlation analyses showed a positive association between consumer and carer attitudes toward \\ medication and between consumer and carer attitudes with adherence. There was a general indication \\ that the greater the difference between consumer and carer attitudes, the lower the level of adherence. \\ Regression analyses revealed that while neither consumer nor carer attitudes were significant predictors \\ of adherence, carer attitudes appeared to have a stronger role in adherence than consumer attitudes. \\ These preliminary results highlight the importance of carer attitudes in relation to patient perceptions and \\ behaviours toward medication, and thus the potential benefits of addressing both consumer and carer \\ attitudes in any intervention for improving adherence.

\section{Disciplines} \\ Education | Social and Behavioral Sciences

\section{Publication Details} \\ Deane, F., McAlpine, E., Byrne, M., Davis, E. \& Mortimer, C. (2018). Are carer attitudes toward medications \\ related to self-reported medication adherence amongst people with mental illness?. Psychiatry Research, \\ 260 158-163.
}




\section{Introduction}

Pharmacotherapy is generally recognised as an important component in the treatment and management of schizophrenia, bipolar, and depression (Barnes and British Assoc, 2011; Cleare et al., 2015; Goodwin et al., 2016). Yet, nonadherence to medication has been reported as greater than 50\% amongst mental health consumers for these disorders (Castle et al., 2012; Ibrahim et al., 2015; Levin et al., 2016; Shafrin et al., 2016). Non-adherence has been defined as clinically significant deviation from the dosage prescribed and exists on a continuum from completely adherent, through levels of partial adherence, to not taking medication at all (Aslani and du Pasquier, 2002). Complete or even partial nonadherence may significantly affect the health and wellbeing of the consumer and their family and has considerable financial implications for the health system (Ascher-Svanum et al., 2006; Gilmer et al., 2004; Hudson et al., 2004; Lacro et al., 2002). Non-adherence in schizophrenia often results in illness exacerbation, relapse, and increased re-hospitalisation (Coombs et al., 2003; Day et al., 2005; Dolder et al., 2003; Kikkert et al., 2008; Lacro et al., 2002; Marland and Cash, 2001; Rettenbacher et al., 2004).

A number of factors have been identified as contributing to medication nonadherence such as psychopathology, cognitive impairment, insight, number and severity of side effects, complexity of dosing pattern, clinician skill, and social support (Fleishchacker et al., 2003). This paper focuses on how consumer and carer attitudes impact adherence.

Previous research has established the importance of subjective attitudes and beliefs of consumers towards their illness and medication in promoting medication 
adherence. Consumers who hold negative beliefs and attitudes towards medication, are less likely to engage in adherence behaviour, while consumers with positive beliefs tend to have higher medication adherence (Lacro et al., 2002; Loffler et al., 2003; Rettenbacher et al., 2004). Rettenbacher et al (2004) found a positive correlation between medication adherence and the consumer's subjective belief as to whether they felt the drug had a positive effect on their illness. It was also found that adherent consumers more often reported the positive effect of medication on their illness and indicated this to be a reason for taking medication in comparison to nonadherent consumers. Similarly, a study among psychiatric consumers in Norway reported that nonadherent consumers felt medication to be less necessary and were more concerned about it than adherent consumers (Jonsdottir et al., 2009).

Less research has examined how the attitudes of individuals such as family members who care for the consumer may shape consumer adherence. Consumer subjective attitudes and beliefs towards medication are developed within an environment in which a consumer is likely to draw on multiple sources of information (Byrne et al., 2008). These sources of information include those significant people around them. In a study of 122 consumers with bipolar disorder more positive medication attitudes were predicted by higher levels of social support and stronger beliefs that their health is influenced by important people in their social environment (Chang et al., 2015).It has been suggested that negative attitudes towards treatment in patients' social environments can have adverse effects on adherence and that it is important to reinforce positive attitudes toward treatment for both patients and relatives (Fleischhacker et al., 2003). Other research has shown an association between carer and consumer attitudes about 
their illness or treatment and found that carer attitudes can impact consumer outcomes. A Latin American study of consumers with schizophrenia and their carers investigated the association between causal belief of schizophrenia with attitudes toward medicine and symptoms (Caqueo-Urizar et al., 2015). Consumer and carer causal beliefs shared small to moderate correlations (e.g., biological causal belief $r=.45$ ). Stronger consumer and carer "magical-religious causal belief" were significantly associated with more negative attitudes toward medicine ( $r=-0.18$ and $\mathrm{r}=-0.13$ respectively). Carers having stronger beliefs in magical-religious causes of mental illness were associated with greater symptom severity in their family member ( $r=0.22$, Caquero-Urizar et al., 2015). A systematic review of medication adherence among Asian Americans with mental illness found that the most successful interventions used psychoeducation that included both consumers and carers, and improved knowledge and attitudes among consumers and family caregivers coincided with clinical improvement (Fancher et al., 2014).

Overall, research has demonstrated the potential impact carers have on consumer attitudes toward medication and the potential effects of consumer and carer beliefs on adherence outcomes. It is important to note that previous research has tended to cast carers and family members in an assisting or supervisory role, but did not consistently and clearly address the attitudinal nature by which this social support might influence consumer adherence behaviour. Moreover, it is not clear from previous research whether it is important that consumer and carer attitudes coincide for optimal adherence or whether discrepancies between attitudes are inconsequential. The current cross-sectional study is exploratory in nature and aims to investigate the relationships between carer and consumer attitudes toward medication and consumer adherence behaviour. Although 
predominantly exploratory, given findings from prior research it was expected that consumer and carer attitudes towards medication and medication adherence would be positively correlated. The study aimed to explore whether consumer and carer attitudes or the discrepancy between these attitudes predicted self-reported medication adherence.

\section{Methods}

\subsection{Procedure}

Eligible consumers were individuals over the age of 18 with a serious mental illness requiring psychotropic medications and eligible carers were individuals over the age of 18 involved in the support of these consumers.

All procedures were reviewed and approved by the relevant Human Research Ethics Committee (HE09/104). The sample was recruited with the support of the carer organisation Association of Relatives and Friends of the Mentally Ill (ARAFMI) in New South Wales (NSW), Australia. Two recruitment pathways were used. Firstly, a researcher attended carer support meetings run by ARAFMI NSW branches at Central Coast, Illawarra and Ryde and invited carers to participate. Secondly, staff at ARAFMI offered participation when carers rang the state head office to make an enquiry. If the carer provided informed consent, they were mailed or emailed a questionnaire pack with coordination of the carer organisation. The pack contained separate information sheets for consumers and carers, separate consumer and carer questionnaires, and reply paid envelopes. All questionnaires were matched with a code to maintain anonymity and returned directly to the researchers via the reply paid envelopes. This was an opportunistic convenience sample and given that staff at the ARAFMI services provided 
information to potential participants we were unable to accurately determine the response rate.

\subsection{Measures}

\subsubsection{Medication adherence}

The Brief Adherence Rating Scale (BARS; Byerly et al., 2008) consists of four items assessing different aspects of adherence:

1. number of prescribed doses per day;

2. how often a dose has been missed over the past month, rated from 1 (less than 7) to 4 (more than 20);

3. how often a consumer took less than prescribed dose over the past month, rated from 1 (76-100\% of the time) to 4 (0-25\% of the time); and

4. overall proportion (0-100\%) of doses taken over the past month.

The last item serves as the final adherence determination (Byerly et al., 2008). In a study with 61 consumers living with schizophrenia, the BARS showed excellent internal consistency ( $\alpha=0.92)$ and good validity, sensitivity (73\%), and specificity (71\%; Byerly et al., 2008).

The Kemp Clinician Rated Compliance Scale (CRCS; Kemp et al., 1996) comprises a single item rating scale in which respondents rate their level of compliance with medication on a seven-point Likert-type scale. The scale points are: 1 (complete refusal), 2 (partial refusal), 3 (reluctant acceptance), 4 (occasional reluctance), 5 (passive acceptance), 6 (moderate participation), and 7 (active participation). The CRCS has been found to be a valid clinician-rated measure in medication adherence studies among 
people with schizophrenia (Kemp et al., 1996; Tsang et al., 2009), and clinician and consumer rated CRCS have shown a medium correlation in a sample of people with schizophrenia $(r=0.50$; Friemann and Wciorka, 2013).The CRCS has shown a small correlation ( $r=0.25$; Kikkert et al., 2008) with the self-reported Medication Adherence Questionnaire (Morisky et al., 1986).

\subsubsection{Attitudes towards medication}

The Drug Attitude Inventory - 10 (DAI-10; Hogan and Awad, 1992) measures satisfaction with medication and understanding of treatment effects. It consists of 10 items that require either an agree/correct (scored 1) or disagree/incorrect (scored -1) response. The final score ranges from -10 to 10 , with the higher the positive or lower the negative score reflecting extent of positivity or negativity towards medications. Internal consistency using Cronbach's alpha in the current study was 0.72 for consumers and 0.78 for carers.

The Beliefs About Medication Questionnaire (BMQ; Horne et al., 1999) - general beliefs subscale contains 8 items that assesses perceptions of the perceived potential for harm and overuse of medication. Items are rated using a 5-point Likert-type scale ranging from 1 (strongly agree) to 5 (strongly disagree). Higher scores represent more negative beliefs. The subscale has demonstrated acceptable internal consistency $(>0.69)$ and validity among people with mental health disorders (Horne et al., 1999; Jonsdottir et al., 2009). Cronbach's alpha in the current study was 0.88 for consumers and 0.85 for carers.

\subsection{Analyses}


Normality plots and statistical tests of normality were inspected. Results showed that the CRCS, BARS and DAI scores did not meet normality assumptions. Therefore we applied the nonparametric tests of Spearman's correlations and bootstrapping for t-tests and regression analyses (5,000 samples).

For all analyses we were interested in examining both consumer and carer reported adherence. This enabled judgments to be made about how results might be impacted by different respondent groups. Also, a composite adherence measure was calculated using standardized scores on the CRCS and BARS4 (overall proportion of doses taken in prior month). The purpose was to maximize use of the adherence data and improve reliability from using single item scores only. The composite scores showed acceptable internal consistency for carers $(\alpha=0.76)$ and marginally acceptable internal consistency for consumers $(\alpha=0.66)$.

Spearman's correlations were used to test the associations between consumer and carer attitudes and adherence. Paired t-tests with bootstrapping were also used to detect any differences between carer and consumer scores on attitudinal and adherence measures. Attitude discrepancy was tested by calculating difference scores between consumer and carer attitude measures (BMQ and DAI). These difference scores were then correlated with consumer and carer rated adherence.

Finally, the prediction of adherence was tested using regression analysis with bootstrapping. Separate multiple regressions with bootstrapping on the consumer and carer adherence composite scores were performed. In order to determine the variables to include in a regression analysis, correlations and $t$-tests were conducted between carer and consumer composite adherences scores with demographic and clinical characteristics 
and both consumer and carer attitude measures. Given that use of difference scores as predictors in multiple regression usually leads to loss of model fit (Edwards, 2001), when a difference score and an individual score from which it was calculated (e.g. BMQ difference score and consumer BMQ score) both correlated significantly with the composite adherence score, we selected the individual scores for the regression.

\section{Results}

\subsection{Sample characteristics}

The sample consisted of 42 carers and 42 of their family members with a mental illness who were receiving treatment (consumers). The mean age of carers was 54.61 $(S D=13.48$, range $=19-78)$ and the majority of carers were female $(64 \%)$. Consumers $(N=42)$ were equally distributed in gender and had a mean age of $41.21(S D=14.63$, range $=18-67)$. Australia was the most frequently reported place of birth for participants with $79 \%$ of carers and $95 \%$ of consumers reported to be born in Australia. As shown in Table 1, the most frequent mental health diagnosis reported was schizophrenia/schizoaffective (43\%), followed by bipolar (26\%) and depression (17\%). Carers were mostly parents (48\%) and the most frequently reported period of care was over 10 years $(57 \%)$.

[INSERT Table 1]

\subsection{Descriptives}

Frequency analysis of the CRCS adherence scale for consumers revealed that 
$50 \%$ reported active participation, while $29 \%$ reported moderate participation. This is consistent with mean consumer BARS4 scores of $91.98 \%(S D=19.05)$ adherence to taking the recommended dosage in the past month. For carers, half $(50 \%)$ reported that consumers had active participation, $19 \%$ moderate participation, and $14 \%$ passive acceptance. This is also largely consistent with mean carer BARS4 scores of $92.26 \%$ $(S D=19.07)$

\subsection{Relationships between consumers and carers medication attitudes and adherence measures}

Consumer and carer reported adherence were strongly related for both the CRCS $(r=0.67, p<0.01)$ and BARS4 $(r=0.61, p<0.01)$ scores. Similarly, there was a strong positive correlation between carer and consumer scores on both the DAI-10 ( $r=0.72$, $p<0.01)$ and BMQ $(r=0.66, p<0.01)$. Table 2 shows the results of paired $t$-tests with bootstrapping (5,000 samples) between consumer and carer adherence and attitude measures. The results indicated no significant differences between consumer and carer reported adherence on the CRCS or BARS4 $(p<0.05)$ or attitudes on the DAI-10 and BMQ $(p<0.05)$.

\section{[INSERT Table 2]}

\subsection{Correlations between consumer and carer attitudes and consumer adherence}

Overall, there was a positive association between consumer and carer attitudes and adherence measures, such that the more positive the attitudes, the greater the 
adherence (see Table 3). This pattern of relationships between attitude measures and adherence generally remains when comparing consumers and carers separately. Of note, specific attitudes seemed to relate more consistently with adherence than general attitudes (BMQ); carer general attitudes did not share a significant relationship with any adherence outcome. Also, the number of days in which less than the prescribed dose was taken (BARS3) was related to consumer attitudes only.

\section{[INSERT Table 3]}

\subsection{Correlations between discrepancy in consumer and carer attitudes with consumer adherence}

Discrepancies in attitudes towards medication were correlated with both consumer and carer ratings of adherence (Table 3). The difference in specific attitudes (DAI-10 diff) was more consistently related to adherence than the difference in general attitudes (BMQ diff), which was related to carer reported CRCS ratings only. The greater the difference between consumer and carer specific attitudes, the lower the level of adherence.

\subsection{Consumer and carer attitudes predicting adherence}

Separate multiple regression analyses with bootstrapping (5,000 samples) were performed on consumer and carer composite adherence scores. Predictors entered for consumer reported adherence were consumer and carer DAI-10 scores. Predictors entered for carer reported adherence were consumer and carer DAI-10 scores and consumer 
BMQ score. Correlations and $t$-tests showed that demographic and clinical characteristics were unrelated to the composite adherence scores (all $p>0.05$ ).

Table 4 provides a summary of the regression results. For consumers, the model accounted for a significant $42 \%$ of the variance in consumer reported adherence $F_{3,41}=14.28, p<0.01$. There were no significant unique predictors, although carer DAI-10 approached significance $(\beta=0.62, p=0.06)$. For carers, the model accounted for a significant $43 \%$ of the variance in carer reported adherence $F_{3,41}=9.56, p<0.01$ ). Likewise, no predictors reached significance, although carer DAI-10 approached significance $(\beta=0.60, p=0.06)$.

\section{[INSERT Table 4]}

\section{Discussion}

As expected, consumer and carer adherence and attitudes towards medication were positively related. The magnitude of the correlation suggests shared attitudes toward medication. This research does not allow us to determine the extent or direction of influence between consumer and carer but the association of attitudes could be a result of a mutual influence process. It is common for carers to simultaneously hold both positive and negative attitudes towards medication which could further influence the attitudes of a family member with mental illness. For example, a European survey of carers of patients with schizophrenia showed that most carers recognized the importance of medication to help patients get better $(76 \%)$ while at the same time believing it damages their general health (67\%; Svettini et al., 2015). Such concerns are legitimate given the heightened 
health risks associated with many antipsychotic medications (e.g., weight gain, new onset diabetes). The majority agreed on the importance of family support to boost adherence (93\%), and acknowledged that treatment adherence was a burden for patients (65\%), with a notable proportion indicating that it was a daily struggle to get patients to take their medication (38\%). Although the current study provides preliminary evidence of the relationship between carer and consumer attitudes towards medications, there is a need for future longitudinal research in order to gain a better understanding of whether attitude transfer between carers and consumers is a bidirectional process. Clarifying the causal direction will help more effectively target interventions designed to improve medication adherence.

Consumer and carer attitudes toward medication were associated with adherence, with more positive attitudes being associated with greater adherence. Specific attitudes (DAI-10) seemed more consistently related with adherence than general attitudes (BMQ). This was particularly for carers where their general attitudes did not relate at all to consumer or carer reported adherence. These initial findings suggest that, specific attitudes regarding how medication affects the consumer personally appear more useful in understanding adherence than more broad or general attitudes. Also, consumer reports of taking less than the prescribed dose (BARS3) were impacted by their own attitudes only.

Partial support was found for the discrepancy in consumer and carer attitude towards medication correlating with adherence. In general, the findings indicated that the greater the difference between consumer and carer attitudes, the lower the level of adherence. The difference in DAI-10 scores was found to more consistently relate to adherence 
outcomes on the CRCS and adherence composite, while the difference in BMQ scores related to carer reported CRCS adherence only. These results point to the importance of including consumers and carers as collaborators in clinical decision making regarding medication. The need to include carers in decisions about medication has been highlighted in a qualitative study of 29 carers in Australia with most indicating that they had been provided with little or no information about antipsychotic medication from treatment teams (Stomski \& Morrison, 2017). Most carers expressed a desire to be involved in medication decision making but typically felt excluded from this process. The authors concluded that "health professionals need to substantially improve the extent to which they collaborate with carers in medication decisions" (Stomski\& Morrison, 2017, p. 7). Toward this end, it may be helpful for treating clinicians to understand and address consumers and carers attitudes and concerns about medication given the relationship between discrepancy in views and adherence.

The regression models found that consumer and carer attitudes accounted for a substantial and significant amount of variance in adherence, but there were no unique predictors, although carer specific attitudes (DAI-10) approached significance and made a relatively strong contribution to the overall model. It is notable that carer specific attitudes appear to have a substantial role in predicting adherence even when consumers' specific and general attitudes are controlled. This result speaks to the importance of carer influence in consumer medication adherence and thus the need to target both carers and consumers with psychoeducational activities to increase adherence.

A number of limitations to this study should be mentioned. This research is specific to an Australian sample, which impacts the generalisability of the results, while the 
relatively small sample size impacts the generalisability of the results. Given the recruitment strategy relied on staff working within the carer support agency an accurate response rate was not able to be determined and leaving the possibility that unknown bias in the sample may have been introduced. In addition, because the sample was recruited through carer support agencies there may be unknown selection effects (e.g., carers with more knowledge of medications). It is also possible that requiring consent from both carer and consumers may result in selection biases (e.g., more cooperative relationships). Since this was a cross-sectional survey causal links between attitudes and medication adherence cannot be determined. A longitudinal design in this research area would be valuable in exploring the dynamic interplay between consumer and carer attitudes and medication adherence. Finally, adherence was self-reported which is subject to a variety of response biases, namely social desirability in the current study. Future research in this area would benefit from incorporating other methodologies to determine medication adherence for corroboration of self-report data, such as electronic monitoring systems. Nonetheless, a strength of this study was the inclusion of multiple attitude and medication adherence measures. In addition, the novel dyad focus on consumer and their carers provided valuable information on the potential mutual influence processes effecting consumer medication adherence.

\subsection{Conclusions}

This preliminary study suggests a strong link between consumer and carer attitudes toward medication and a positive association between both consumer and carer attitudes with consumer adherence. Moreover, regression analyses showed that while 
neither consumer nor carer attitudes were significant unique predictors of adherence, carer attitudes may play as strong or stronger role in adherence than consumers' attitudes. In addition, there was a general indication that the greater the difference between consumer and carer attitudes, the lower the level of adherence. These initial findings need to be extended in larger more representative samples, but they offer tantalizing possibilities should they be replicated in future research. Specifically, they offer opportunities for the development of interventions that target carer and consumer attitudes toward medication, with the potential of improving consumer medication adherence. 


\section{Conflicts of interest}

None.

\section{Funding}

This research did not receive any specific grant from funding agencies in the public, commercial, or not-for-profit sectors. 


\section{References}

Ascher-Svanum, H., Faries, D.E., Zhu, B., Ernst, F.R., Swartz, M.S., Swanson, J.W., 2006. Medication adherence and long-term functional outcomes in the treatment of schizophrenia in usual care. J Clin Psychiatry 67 (3), 453-460.

Aslani, P., du Pasquier, S., 2002. Compliance, adherence or concordance? Australian Pharmasicist 12 (3), 170-117.

Barnes, T.R.E., British Assoc, P., 2011. Evidence-based guidelines for the pharmacological treatment of schizophrenia: recommendations from the British Association for Psychopharmacology. Journal of Psychopharmacology 25 (5), $567-620$.

Byerly, M.J., Nakonezny, P.A., Rush, A.J., 2008. The Brief Adherence Rating Scale (BARS) validated against electronic monitoring in assessing the antipsychotic medication adherence of outpatients with schizophrenia and schizoaffective disorder. Schizophr Res 100 (1-3), 60-69.

Byrne, M.K., Deane, F.P., Caputi, P., 2008. Mental health clinicians' beliefs about medicines, attitudes, and expectations of improved medication adherence in patients. Eval Health Prof 31 (4), 390-403.

Caqueo-Urizar, A., Boyer, L., Baumstarck, K., Gilman, S.E., 2015. The relationships between patients' and caregivers' beliefs about the causes of schizophrenia and clinical outcomes in Latin American countries. Psychiatry Res 229 (1-2), 440446.

Castle, T., Cunningham, M.A., Marsh, G.M., 2012. Antidepressant medication adherence via interactive voice response telephone calls. Am J Manag Care 18 (9). 
Chang, C.W., Sajatovic, M., Tatsuoka, C., 2015. Correlates of attitudes towards mood stabilizers in individuals with bipolar disorder. Bipolar Disorders 17 (1), 106-112.

Cleare, A., Pariante, C.M., Young, A.H., Anderson, I.M., Christmas, D., Cowen, P.J., et al., 2015. Evidence-based guidelines for treating depressive disorders with antidepressants: A revision of the 2008 British Association for Psychopharmacology guidelines. Journal of Psychopharmacology 29 (5), 459525.

Coombs, T., Deane, F.P., Lambert, G., Griffiths, R., 2003. What influences patients' medication adherence? Mental health nurse perspectives and a need for education and training. Int J Ment Health Nurs 12 (2), 148-152.

Day, J.C., Bentall, R.P., Roberts, C., Randall, F., Rogers, A., Cattell, D., et al., 2005. Attitudes toward antipsychotic medication - The impact of clinical variables and relationships with health professionals. Arch Gen Psychiatry 62 (7), 717-724.

Dolder, C.R., Lacro, J.P., Jeste, D.V., 2003. Adherence to antipsychotic and nonpsychiatric medications in middle-aged and older patients with psychotic disorders. Psychosom Med 65 (1), 156-162.

Fancher, T.L., Lee, D., Cheng, J.K.Y., Yang, M.S., Yang, L., 2014. Interventions to improve adherence to psychotropic medication in clients of Asian descent: a systematic review. Asian Am J Psychol 5 (1), 22-34.

Fleischhacker, W.W., Oehl, M.A., Hummer, M., 2003. Factors influencing compliance in schizophrenia patients. J Clin Psychiatry 64, 10-13. 
Friemann, K., Wciorka, J., 2013. Four measures of treatment compliance among patients recovering from psychotic episodes - a comparative study. Psychiatr Pol 47 (5), 759-772.

Gilmer, T.P., Dolder, C.R., Lacro, J.P., Folsom, D.P., Lindamer, L., Garcia, P., et al., 2004. Adherence to treatment with antipsychotic medication and health care costs among Medicaid beneficiaries with schizophrenia. Am J Psychiatry 161 (4), 692699.

Goodwin, G.M., Haddad, P.M., Ferrier, I.N., Aronson, J.K., Barnes, T.R.H., Cipriani, A., et al., 2016. Evidence-based guidelines for treating bipolar disorder: Revised third edition recommendations from the British Association for Psychopharmacology. Journal of Psychopharmacology 30 (6), 495-553.

Hogan, T.P., Awad, A.G., 1992. Subjective response to neuroleptics and outcome in schizophrenia: a re-examination comparing two measures. Psychol Med 22 (2), $347-352$.

Horne, R., Weinman, J., Hankins, M., 1999. The beliefs about medicines questionnaire: The development and evaluation of a new method for assessing the cognitive representation of medication. Psychol Health 14 (1), 1-24.

Hudson, T.J., Owen, R.R., Thrush, C.R., Han, X.T., Pyne, J.M., Thapa, P., et al., 2004. A pilot study of barriers to medication adherence in schizophrenia. J Clin Psychiatry 65 (2), 211-216.

Ibrahim, A.W., Yahya, S., Pindar, S.K., Wakil, M.A., Garkuwa, A., Sale, S., 2015. Prevalence and predictors of sub-optimal medication adherence among patients 
with severe mental illnesses in a tertiary psychiatric facility in Maiduguri, Northeastern Nigeria. Pan Afr Med J 21.

Jonsdottir, H., Friis, S., Horne, R., Pettersen, K.I., Reikvam, A., Andreassen, O.A., 2009. Beliefs about medications: measurement and relationship to adherence in patients with severe mental disorders. Acta Psychiatr Scand 119 (1), 78-84.

Kemp, R., Hayward, P., Applewhaite, G., Everitt, B., David, A., 1996. Compliance therapy in psychotic patients: Randomised controlled trial. Br Med J 312 (7027), 345-349.

Kikkert, M.J., Barbui, C., Koeter, M.W.J., David, A.S., Leese, M., Tansella, M., et al., 2008. Assessment of medication adherence in patients with schizophrenia. J Nerv Ment Dis 196 (4), 274-281.

Lacro, J.P., Dunn, L.B., Dolder, C.R., Leckband, S.G., Jeste, D.V., 2002. Prevalence of and risk factors for medication nonadherence in patients with schizophrenia: A comprehensive review of recent literature. J Clin Psychiatry 63 (10), 892-909.

Levin, J.B., Aebi, M.E., Tatsuoka, C., Cassidy, K.A., Sajatovic, M., 2016. Adherence to psychotropic and nonpsychotropic medication among patients with bipolar disorder and general medical conditions. Psychiatr Serv 67 (3), 342-345.

Loffler, W., Kilian, R., Toumi, M., Angermeyer, M.C., 2003. Schizophrenic patients' subjective reasons for compliance and noncompliance with neuroleptic treatment. Pharmacopsychiatry 36 (3), 105-+.

Marland, G.R., Cash, K., 2001. Long-term illness and patterns of medicine taking: are people with schizophrenia a unique group? J Psychiatr Ment Health Nurs 8 (3), 197-204. 
Morisky, D.E., Green, L.W., Levine, D.M., 1986. Concurrent and predictive validity of a self-reported measure of medication adherence Med Care 24 (1), 67-74.

Rettenbacher, M.A., Hofer, A., Eder, U., Hummer, M., Kemmler, G., Weiss, E.M., et al., 2004. Compliance in schizophrenia: psychopathology, side effects, and patients' attitudes toward the illness and medication. J Clin Psychiatry 65 (9), 1211-1218.

Shafrin, J., Schwartz, T.T., Lakdawalla, D.N., Forma, F.M., 2016. Estimating the value of new technologies that provide more accurate drug adherence information to providers for their patients with schizophrenia. Journal of Managed Care \& Specialty Pharmacy 22 (11), 1285-1291.

Stomski, N.J., Morrison, P., 2017. Carer's involvement in decision making about antipsychotic medication: A qualitative study. Health Expect, Epub 1-7.

Svettini, A., Johnson, B., Magro, C., Saunders, J., Jones, K., Silk, S., et al., 2015. Schizophrenia through the carers' eyes: results of a European cross-sectional survey. J Psychiatr Ment Health Nurs 22 (7), 472-483.

Tsang, H.W.H., Fung, K.M.T., Corrigan, P.W., 2009. Psychosocial and sociodemographic correlates of medication compliance among people with schizophrenia. J Behav Ther Exp Psychiatry 40 (1), 3-14. 
Table 1. Demographics and clinical characteristics of consumers $(N=42)$ and carers $(N=42)$.

\begin{tabular}{|c|c|c|}
\hline & Frequency & Percent \\
\hline \multicolumn{3}{|l|}{ Mental health diagnosis } \\
\hline Schizophrenia/ Schizoaffective & 18 & 43 \\
\hline Bipolar & 11 & 26 \\
\hline Depression & 7 & 17 \\
\hline Other & 6 & 14 \\
\hline \multicolumn{3}{|l|}{ Medication type } \\
\hline Antipsychotic & 29 & 69 \\
\hline Antidepressant & 7 & 17 \\
\hline Not stated & 4 & 10 \\
\hline Other & 2 & 5 \\
\hline \multicolumn{3}{|l|}{ Treatment length } \\
\hline $0-2$ years & 1 & 2 \\
\hline $3-5$ years & 13 & 29 \\
\hline $6-9$ years & 8 & 18 \\
\hline 10 years and over & 23 & 51 \\
\hline
\end{tabular}


Years as carer

0-2 years

2

5

3-5 years

8

19

6-9 years

8

19

10 years and over

24

57

Carer relationship with consumer

Parent

20

48

Partner

15

36

Child

6

14

Friend

1

2

Note. Percentages do not always add to 100 due to rounding. 
Table 2. Comparison of mean attitude and adherence scores for consumers and carers.

\begin{tabular}{lllll}
\hline & Consumer $M(S D)$ & Carer $M(S D)$ & $t$-value & Boot 95 CI \\
\hline DAI-10 & $3.51(5.08)$ & $4.13(4.98)$ & 0.41 & $-0.71,1.29$ \\
BMQ & $2.81(.91)$ & $2.59(.82)$ & -1.57 & $-0.41,0.04$ \\
CRCS & $6.02(1.56)$ & $5.74(1.65)$ & -1.57 & $-0.55,0.05$ \\
BARS4 & $88.84(24.12)$ & $91.22(19.57)$ & 0.23 & $-2.12,2.69$ \\
Adherence & $-0.02(.89)$ & $-0.02(.93)$ & -0.58 & $-0.16,0.07$ \\
composite & & & & \\
\hline
\end{tabular}

Note 1. DAI-10=Drug Attitude Inventory - 10; BMQ=Beliefs About Medication

Questionnaire; CRCS=Clinician Rated Compliance Scale; BARS4= Brief Adherence

Rating Scale item 4 about the proportion of doses taken.

Note 2 . Boot $95 \mathrm{CI}=$ bootstrap $95 \%$ confidence intervals. 
Table 3. Correlations between consumer and carer attitude and adherence measures.

\begin{tabular}{|c|c|c|c|c|c|c|c|c|c|c|}
\hline & \multicolumn{5}{|c|}{ Consumer } & \multicolumn{5}{|c|}{ Carer } \\
\hline & & & & & Adherence & & & & & Adherence \\
\hline Consumer & CRCS & BARS2 & BARS3 & BARS4 & composite & CRCS & BARS2 & BARS3 & BARS4 & composite \\
\hline DAI-10 & 0.28 & $-0.34 *$ & $0.31 *$ & $0.36^{*}$ & $0.34 *$ & $0.44 * *$ & $-0.44 * *$ & 0.22 & $0.34 *$ & $0.47 * *$ \\
\hline BMQ & -0.29 & $0.42 *$ & $-0.32 *$ & -0.14 & -0.26 & $-0.51 * *$ & $0.49 * *$ & -0.20 & -0.13 & $-0.45^{* *}$ \\
\hline \multicolumn{11}{|l|}{ Carer } \\
\hline DAI-10 & $0.46 * *$ & $-0.39 * *$ & 0.22 & $0.37 *$ & $0.49 * *$ & $0.62 * *$ & $-0.50 * *$ & 0.18 & 0.25 & $0.59 * *$ \\
\hline BMQ & -0.15 & 0.20 & -0.12 & -0.17 & -0.18 & -0.25 & 0.30 & -0.02 & -0.09 & -0.24 \\
\hline \multicolumn{11}{|l|}{ Difference } \\
\hline \multicolumn{11}{|l|}{ scores } \\
\hline DAI-10 diff & $0.45^{* *}$ & -0.27 & 0.04 & 0.08 & $0.35^{*}$ & $0.43 *$ & -0.20 & 0.02 & -0.09 & $0.32 *$ \\
\hline BMQ diff & 0.16 & -0.19 & 0.23 & 0.08 & 0.09 & $0.32 *$ & -0.23 & 0.19 & 0.09 & 0.23 \\
\hline
\end{tabular}


$* p<0.05, * * p<0.01$

Note . RCS=Clinician Rated Compliance Scale; BARS2= Brief Adherence Rating Scale item 2 about number of days did not take medication; BARS3= Brief Adherence Rating Scale item 3 about number of days took less than prescribed number; BARS4= Brief Adherence Rating Scale item 4 about the proportion of doses taken; DAI-10 diff=difference score between consumer and carer Drug Attitude Inventory - 10 (DAI-10) ratings; BMQ diff= difference score between consumer and carer Beliefs About Medication Questionnaire (BMQ) ratings. 
Table 4. Regression on consumer and carer standardized adherence scores.

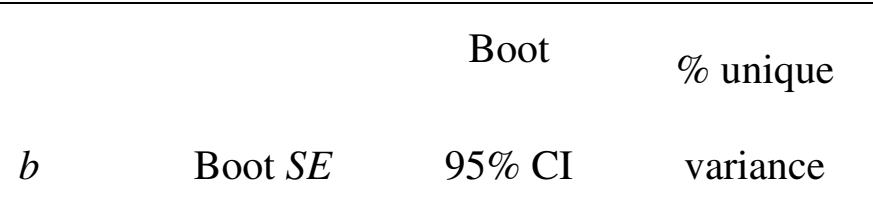

Consumer adherence

scores

$\begin{array}{lcccc}\text { Consumer DAI-10 } & 0.01 & 0.03 & -0.07,0.06 & 0.09 \\ \text { Carer DAI-10 } & 0.10 & 0.04 & 0.02,0.19 & 16.24\end{array}$

Carer adherence scores

$\begin{array}{lllll}\text { Consumer DAI-10 } & -0.01 & 0.03 & -0.09,0.04 & 0.07\end{array}$

$\begin{array}{lllll}\text { Consumer BMQ } & -0.13 & 0.16 & -0.45,0.17 & 0.64\end{array}$

$\begin{array}{lllll}\text { Carer DAI-10 } & 0.11 & 0.04 & 0.03,0.20 & 14.75\end{array}$

$* p<0.05, * * p<0.01$

Note 1. DAI-10=Drug Attitude Inventory $-10 ; \mathrm{BMQ}=$ Beliefs About Medication Questionnaire.

Note 2. $b=$ unstandardised regression coefficient; Boot $S E=$ bootstrap standard error; Boot $95 \mathrm{CI}=$ bootstrap $95 \%$ confidence intervals; $\%$ unique variance $=$ percentage of unique variance accounted for in the adherence score, as indicated by the squared semipartial correlation coefficient. 